Observati on of the "Self-Heal ing" of an Error Fi el d I sl and in the Large Hel i cal Devi ce

\begin{tabular}{|l|l|}
\hline $\begin{array}{l}\text { j our nal or } \\
\text { publ i cat i on } \mathrm{titl} \text { e }\end{array}$ & Physi cal Revi ew Let ter s \\
\hline vol une & Vol .87 \\
\hline number & I ssue 13 \\
\hline page $\mathrm{r}$ ange & 135002-1 135002-4 \\
\hline year & 2001- 09-01 \\
\hline URL & ht t p: //hdl . handl e. net /10655/2287 \\
\hline
\end{tabular}




\title{
Observation of the "Self-Healing" of an Error Field Island in the Large Helical Device
}

\author{
K. Narihara, ${ }^{1}$ K. Y. Watanabe,${ }^{1}$ I. Yamada, ${ }^{1}$ T. Morisaki, ${ }^{1}$ K. Tanaka, ${ }^{1}$ S. Sakakibara,${ }^{1}$ K. Ida, ${ }^{1}$ R. Sakamoto, ${ }^{1}$ \\ N. Ohyabu, ${ }^{1}$ N. Ashikawa, ${ }^{2}$ M. Emoto, ${ }^{1}$ H. Funaba, ${ }^{1}$ M. Goto, ${ }^{1}$ H. Hayashi, ${ }^{1}$ H. Idei, ${ }^{1}$ K. Ikeda, ${ }^{1}$ S. Inagaki, ${ }^{1}$ \\ N. Inoue,${ }^{1}$ O. Kaneko, ${ }^{1}$ K. Kawahata,${ }^{1}$ T. Kobuchi, ${ }^{2}$ A. Komori, ${ }^{1}$ S. Kubo, ${ }^{1}$ R. Kumazawa, ${ }^{1}$ S. Masuzaki, ${ }^{1}$ \\ J. Miyazawa, ${ }^{1}$ S. Morita, ${ }^{1}$ O. Motojima, ${ }^{1}$ S. Murakami, ${ }^{1}$ S. Muto, ${ }^{1}$ T. Mutoh, ${ }^{1}$ Y. Nagayama,${ }^{1}$ Y. Nakamura, ${ }^{1}$ \\ H. Nakanishi, ${ }^{1}$ K. Nishimura, ${ }^{1}$ N. Noda,${ }^{1}$ T. Notake,${ }^{3}$ S. Ohdachi, ${ }^{1}$ Y. Oka, ${ }^{1}$ K. Ohkubo, ${ }^{1}$ M. Osakabe, ${ }^{1}$ S. Ozaki, ${ }^{1}$ \\ B. J. Peterson, ${ }^{1}$ A. Sagara, ${ }^{1}$ K. Saito,${ }^{3}$ H. Sasao, ${ }^{2}$ M. Sasao, ${ }^{1}$ K. Sato, ${ }^{1}$ M. Sato,${ }^{1}$ T. Seki, ${ }^{1}$ T. Shimozuma,${ }^{1}$ C. Shoji,${ }^{1}$ \\ S. Sudo, ${ }^{1}$ H. Suzuki, ${ }^{1}$ A. Takayama, ${ }^{1}$ M. Takechi, ${ }^{3}$ Y. Takeiri, ${ }^{1}$ N. Tamura, ${ }^{2}$ K. Toi, ${ }^{1}$ N. Tokuzawa, ${ }^{1}$ Y. Torii, ${ }^{3}$ \\ K. Tsumori, ${ }^{1}$ T. Watari, ${ }^{1}$ H. Yamada,${ }^{1}$ S. Yamaguchi, ${ }^{1}$ S. Yamamoto, ${ }^{3}$ K. Yamazaki, ${ }^{1}$ and Y. Yoshimura ${ }^{1}$ \\ ${ }^{1}$ National Institute for Fusion Science, Toki, 509-5292, Japan \\ ${ }^{2}$ Graduate University for Advanced Studies, Hayama, 240-0193, Japan \\ ${ }^{3}$ Department of Energy Engineering and Science, Nagoya University, Nagoya, 464-8603, Japan
}

(Received 12 March 2001; published 7 September 2001)

\begin{abstract}
It was observed that the vacuum magnetic island produced by an external error magnetic field in the large helical device shrank in the presence of plasma. This was evidenced by the disappearance of flat regions in the electron temperature profile obtained by Thomson scattering. This island behavior depended on the magnetic configuration in which the plasmas were produced.
\end{abstract}

PACS numbers: $52.55 . \mathrm{Hc}$

Existence of a set of nested magnetic surfaces (magnetic configuration) is a prerequisite for almost all toroidal magnetic confinement devices [1]. In the tokamak, a magnetic configuration is generated by plasma current plus external coil currents, and hence the concept of a vacuum magnetic configuration does not exist. On the other hand, the stellarator, by its definition, possesses a vacuum magnetic configuration that is able to confine plasma just as Nature possesses a vacuum space-time that allows the existence of matter. This fact makes it easier in the stellarator than in the tokamak to study the interactions between the magnetic configuration and plasma such as the formation of topological defects and their reactions on the plasma behavior. The vacuum magnetic configuration of a stellarator is inevitably accompanied by topological defects such as magnetic islands and stochastic regions as a result of a weak violation of a helical symmetry. If these defects are large in size or numerous, they will deteriorate the plasma confinement considerably. This was a central issue in stellarator development. However, nowadays, we can make these topological defects small enough not to degrade the plasma confinement over a wide region by optimizing the coil-winding law [2]. Although the vacuum magnetic configuration problem was thus solved, the question whether a fairly good magnetic configuration is preserved in the presence of plasma has not yet been settled. Cary and Kotschenreuther [3] showed that sharply peaked currents near the island enhance or limit the island size, depending on whether the average curvature is bad or good. They also showed that in the hill region the islands overlap for arbitrary small beta $\beta=$ (kinetic pressure/magnetic pressure). Hegna and Bhattacharjee [4] generalized the above statement for higher $\beta$ plasma with the result that the well/hill criterion should be replaced by the resistive interchange stability criterion. Hayashi et al. [5] computationally found that the vacuum magnetic surfaces of an $L=2 / M=10$ heliotron/torsatron configuration are, indeed, broken at high $\beta$, giving a more stringent limit on $\beta$ than that imposed by equilibrium. Hayashi et al. [6] also discovered that as $\beta$ increases the islands present in the vacuum heliac configuration diminish ("self-heal") and then reappear with the flipped phase at a higher $\beta$. Bhattacharjee et al. [7] formulated a theory to explain the above "self-healing" phenomenon. Hegna [8] further generalized the above theories to incorporate the effect of a bootstrap current. Since these theoretical and numerical predictions have a crucial consequence for stellarator development, it is quite important to give experimental evidence for/against the predictions. Although there were many measurements of the vacuum islands in stellarators [9], no report appeared on their behavior in plasmas. This is partly due to the lack of appropriate diagnostics, and partly due to the smallness of the hitherto used stellarators. With the start of the large helical device (LHD) experiment with sophisticated diagnostics [10], we could first (we think) observe the island behavior in a stellarator in the presence of plasma.

LHD $[11,12]$ is a superconducting magnet system composed of an $L=2 / M=10$ helical coil and three pairs of poloidal coils. The currents in these coils are fed independently, allowing us to change the shape and position of the magnetic surfaces as well as the magnetic field strength on axis $B_{\text {ax }}$ up to $3 \mathrm{~T}$. The plasmas we analyze in this Letter were produced in two magnetic configurations with different magnetic axis positions: $R_{\mathrm{ax}}=3.75$ and $3.6 \mathrm{~m}$. The poloidal cross sectional views of magnetic field lines at a horizontally elongated section are shown for the $R_{\mathrm{ax}}=3.6$ and $3.75 \mathrm{~m}$ configurations in Figs. 1(A) and 


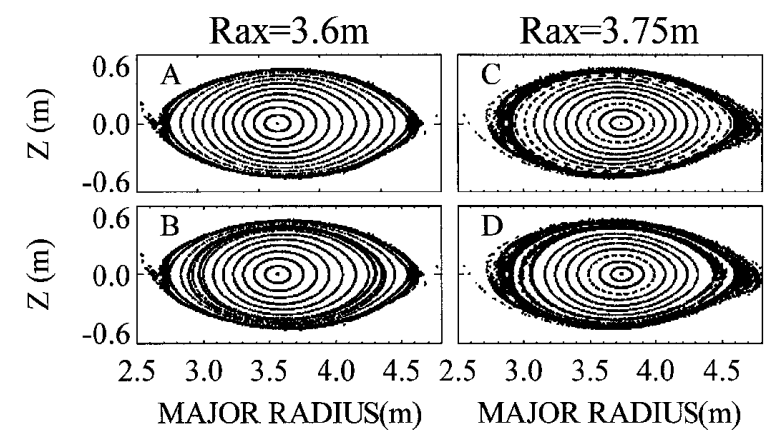

FIG. 1. Poloical cross sectional views of a bundle of magnetic field lines in LHD. Top: the designed (A) $R_{\mathrm{ax}}=3.6 \mathrm{~m}$ and (C) $R_{\mathrm{ax}}=3.75 \mathrm{~m}$ configurations. Bottom: the error field added (B) $R_{\mathrm{ax}}=3.6 \mathrm{~m}$ and (D) $R_{\mathrm{ax}}=3.75 \mathrm{~m}$ configurations. Note the magnetic islands in (B) and (D) with the error fields.

$1(C)$, respectively. In the absence of plasma (vacuum), a substantially wide space is filled with almost completely nested magnetic surfaces bounded by a stochastic region. It is noted that the $R_{\mathrm{ax}}=3.6 \mathrm{~m}$ configuration has a much thinner stochastic region than the $R_{\mathrm{ax}}=3.75 \mathrm{~m}$ configuration. The above two configurations are ideal ones that would be obtained if the construction were perfect. The practically obtained magnetic field would contain small error fields arising from sources such as coil-winding/positioning errors. The error field may resonate on the low order rational magnetic surfaces and may generate magnetic islands. Indeed, a vacuum error field island was observed by an electron beam mapping method [12] to have a mode $m / n=1 / 1$ and the $O$-point crossing width of $80 \mathrm{~mm}$ at a vertically elongated section. The $R_{\mathrm{ax}}=3.6$ and $3.75 \mathrm{~m}$ configurations with the error field added give the cross sectional views as shown in Figs. 1(B) and 1(D), respectively, at the toroidal position where the Thomson scattering measurements were done. Our interest is toward what happens to these vacuum magnetic configurations when plasma is produced. Although the exact magnetic configuration is difficult to measure directly with the presently available diagnostics, we may infer the state of a magnetic configuration from the fine-spatial profile of electron temperature $\left(T_{e}\right)$, which is supposed to be constant along a magnetic field line, as was demonstrated in tokamaks $[13,14]$ : If a $T_{e}$ profile has a flat region or a bump near a rational surface, this probably implies an island, though the possibility of a very local heating or a local transport anomaly cannot be ruled out. The Thomson scattering system [15] installed on LHD was designed to repetitively measure $T_{e}$ and electron density $\left(n_{e}\right)$ at 200 positions along the major radius on the $Z=0$ plane at the horizontally elongated section. At present, significant signals are observed only on 120 positions. The spatial resolution, defined by the full width at half maximum of the position-responsivity relation of the light collection optics, is $20 \mathrm{~mm}$ at the scattering position $R=4.5 \mathrm{~m}$ and $35 \mathrm{~mm}$ at $R=2.5 \mathrm{~m}$.

In the static magnetic configurations shown in Fig. 1, prefilled hydrogen or helium gases were ionized by sub-
MW range electron cyclotron resonance waves and subsequently heated and maintained by neutral beam injection (NBI) (150 keV, $\sim 5 \mathrm{MW})$. Normally, repeated gas puffing or multiple hydrogen ice pellet injection fueled the plasma.

The evolution of the island was best observed in the $R_{\mathrm{ax}}=3.6 \mathrm{~m}$ configuration. An example is shown in Fig. 2, together with the time evolutions of the diamagnetic energy $W_{p}$ and the line averaged electron density $\left\langle n_{e}\right\rangle$. Figure 2(A): when $\left\langle n_{e}\right\rangle$ rose enough to yield Thomson scattering signals, $t=0.7 \mathrm{~s}$, the width of flat regions just inside the $\iota / 2 \pi=1$ surface, which are supposed to represent an island, were already smaller than the vacuum island size. In this low density phase, the island can be noticed only when several laser-shot data are averaged. The reduced island changed its size from time to time until $t \sim 1.5 \mathrm{~s}$. Figure 2(B): in the period $1.6 \mathrm{~s}<t<2.6 \mathrm{~s}$, the island almost disappeared. Figure 2(C): in the rapidly decaying phase, when $\left\langle n_{e}\right\rangle$ is high and $T_{e}$ is low and therefore the plasma is highly collisional, the island reappeared with the width and phase consistent with the vacuum island. In the $R_{\mathrm{ax}}=3.75 \mathrm{~m}$ configuration, the island seemed to shrink more easily than in the $R_{\mathrm{ax}}=3.6 \mathrm{~m}$ configuration, as shown in Fig. 3. When $\left\langle n_{e}\right\rangle$ rose enough to yield Thomson scattering signals [Fig. 3(A)], the island already shrank almost completely, and seldom reappeared in the rapidly decaying phase of the plasma [Fig. 3(C)]. We notice weak bends in the $T_{e}$ profiles around the $\iota / 2 \pi=1$ positions at both sides.

All the above data were obtained for the plasma in which the working gas was fueled gradually by gas puffing. When multiple hydrogen ice pellets were injected, the reduced island grew drastically, as in the example shown in Fig. 4. Upon pellet injection, the almost "self-healed"

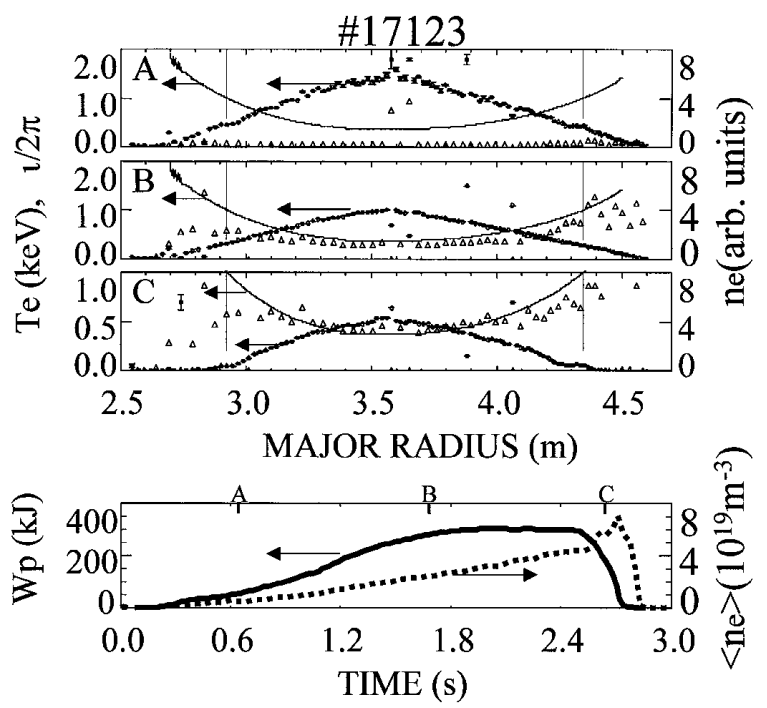

FIG. 2. $\quad T_{e}(\bigcirc)$ and $n_{e}(\triangle)$ profiles at three different instances of a discharge in the $R_{\mathrm{ax}}=3.6 \mathrm{~m}$ configuration. $B_{\mathrm{ax}}=2.75 \mathrm{~T}$. The solid lines are $\iota / 2 \pi$ profiles. The vertical lines show $\iota / 2 \pi=1$ positions. Diamagnetic energy $W_{p}$ and line averaged electron density $\left\langle n_{e}\right\rangle$ evolved as shown in the bottom. 


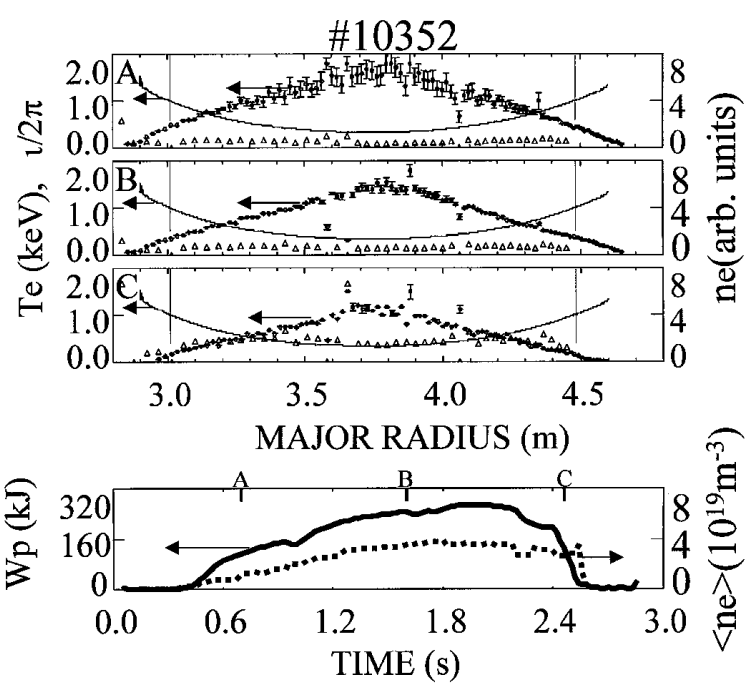

FIG. 3. Same as Fig. 2 for a discharge in the $R_{\mathrm{ax}}=3.75 \mathrm{~m}$ configuration with $B_{\mathrm{ax}}=2.5 \mathrm{~T}$.

island reappeared [Figs. 4(A) and 4(B)]. Interestingly, just after pellet injection [Fig. 4(B)] the phase flipped by $\pi / 2$ and gradually returned to the vacuum phase. The island size at $t=1.6 \mathrm{~s}$ [Fig. 4(C)] is larger than the vacuum island. In addition to the above typical behavior, the island showed diverse behavior on pellet injection: In some discharges the island grew with the same phase as the vacuum island, and in others it preserved its "self-healed" state.

All the data shown above were obtained for low $\beta$ plasma. A natural question is what happens when $\beta$ is further raised. The $T_{e}$ profile of a plasma with the average $\beta \sim 2 \%$ is shown in Fig. 5. Although the central region somewhat became irregular, the $1 / 1$ island remains incompletely "self-healed." Furthermore, the plasma became larger, exceeding the vacuum stochastic region, implying a mechanism to "self-heal" even the stochastic region.
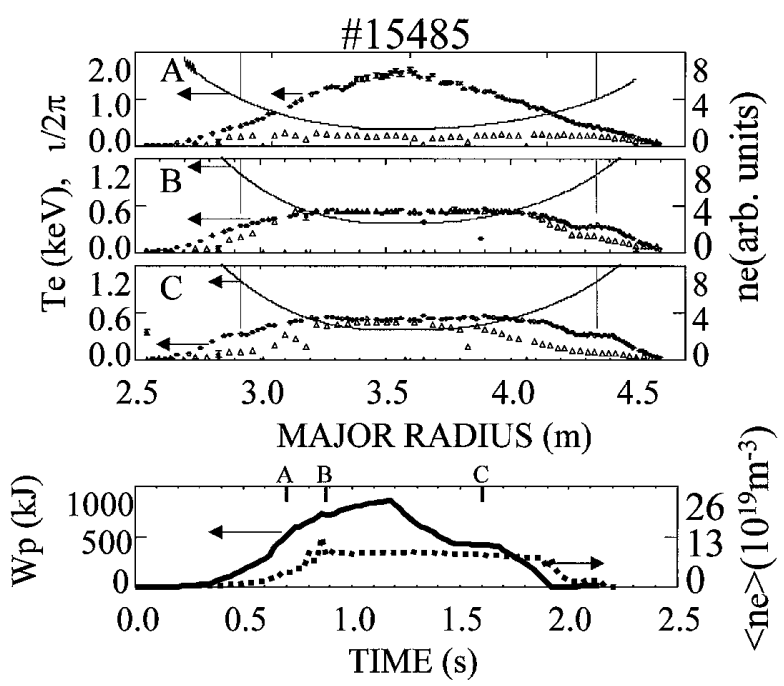

FIG. 4. Same as Fig. 2 for a discharge in the $R_{\mathrm{ax}}=3.6 \mathrm{~m}$ configuration with five pellets injected between $0.6-0.8 \mathrm{~s} . B_{\mathrm{ax}}=$ $2.75 \mathrm{~T}$.
The change in the vacuum magnetic island implies that currents were induced in the plasma or the surrounding conductor. If the current is driven by an internal force that does not interact with (is not aware of) the island, the resultant internal error field will have a phase with respect to the external error field that distributes as $\phi_{0}=$ \{const $\left.+2 \pi / 10^{*} k, k=0,9\right\}$ with an equal probability of occurrence because LHD is invariant under the rotation angle $\left\{2 \pi / 10^{*} k, k=0,9\right\}$ about the central axis $\left(C_{10}\right.$ symmetry). The interference between the internal and external error fields will cause the island to show up with different shapes and phases shot by shot, which was observed only for pellet injection. The NBI, whose operation breaks the $C_{10}$ symmetry, may generate an internal error field with the phase that happens to cancel the external error field. We examined $T_{e}$ profiles of plasmas heated and maintained by NBI with co-, counter-, and balanced injection modes, but no appreciable difference was found. Hence, the island shrinkage can be ascribed to a plasma current that interacts with the island itself.

It may be worthy to compare the above observations with the theory given by Hegna [8], though its applicability to the $n / m=1 / 1$ island is somewhat questionable. The saturated island width in units of toroidal flux $\Psi$ at a rational surface is given by

$$
W=D / 2+\left(D^{2} / 4+\left|C+s w_{v}^{2}\right|\right)^{0.5},
$$

where $s= \pm 1$ depending on whether the phase difference between the internal and external error fields $\phi_{B}=0$ or $\pi$, and $w_{v}$ is the width of the vacuum island. $C$ comes from the field produced by the resonant component of the Pfirsch-Schulter (PS) current, which can be regarded as the internal error field and is absent in the tokamak because of its axisymmetry. $D$ arises from the field produced by the currents that interact nonlinearly with the island. Hegna included the bootstrap current as well as the PS current and expressed $D=c 1^{*}\left(D_{\mathrm{nc}}+D_{R}^{\dagger}\right)$ with positive constants $c 1$ of $O(1)$, where $D_{\text {nc }}$ is the bootstrap current contribution (the neoclassical effect) and $D_{R}{ }^{\dagger}$ is the PS current contribution (the Glasser effect). If $D<0$, Eq. (1) implies the shrinkage of the island. Following the

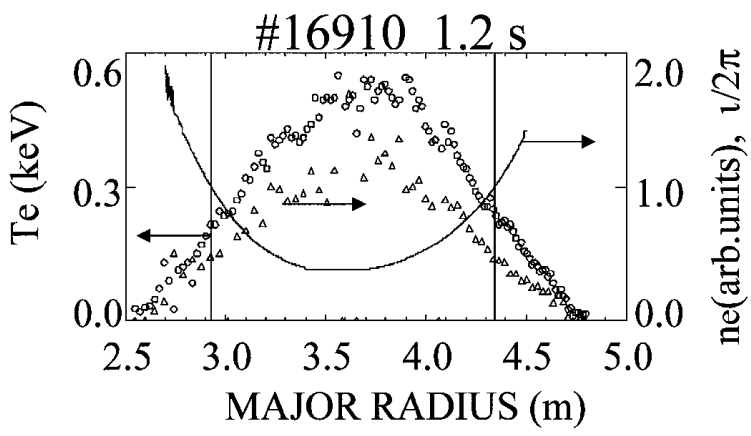

FIG. 5. $T_{e}$ and $n_{e}$ profiles of a $\beta \sim 2 \%$ plasma in the $R_{\mathrm{ax}}=$ $3.6 \mathrm{~m}$ configuration. $B_{\mathrm{ax}}=0.75 \mathrm{~T}$. 


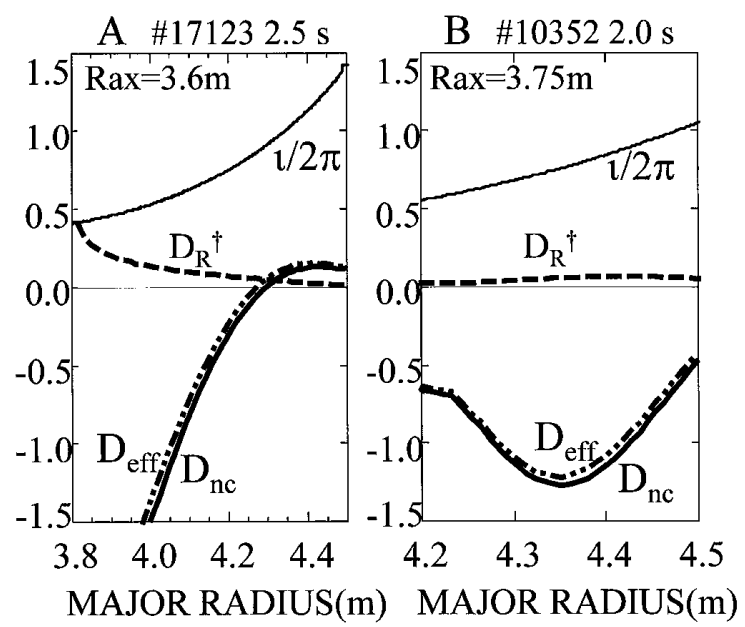

FIG. 6. Resistive interchange effect $D_{R}^{\dagger}$ and neoclassical effect $D_{\text {nc }}$ for the plasma shown in Fig. 2(B) (left) and the plasma shown in Fig. 3(B) (right).

expressions given in [8], we numerically calculated $D_{\mathrm{nc}}$ and $D_{R}{ }^{\dagger}$ for the plasmas formed in the $R_{\mathrm{ax}}=3.6$ and $3.75 \mathrm{~m}$ configurations with the results shown in Figs. 6(A) and 6(B), respectively. For both configurations, $D_{R}{ }^{\dagger}$ is slightly positive and then has a weakly destabilizing effect. The iota profile has a positive radial gradient for both configurations, which together with a negative pressure gradient satisfies the conventional stability condition for the neoclassical tearing mode. However, the situation is somewhat more complicated for the LHD case. The radial dependence of the magnetic field strength spectrum for the $R_{\mathrm{ax}}=3.6 \mathrm{~m}$ configuration makes $D_{\mathrm{nc}}$ slightly positive in the outer region $(R>4.3 \mathrm{~m})$, as shown in Fig. 6(A) [16]. The overall effect $D_{\text {eff }}=D_{\text {nc }}+D_{R}{ }^{\dagger}$ is slightly positive at about $\iota / 2 \pi=1$, implying the growth of the island. However, in view of the limited accuracy of the calculation [16] and the crudeness of the $n_{e}$ profile, the obtained small $D_{\text {eff }}$ should be regarded as rather marginal. On the other hand, for the $R_{\mathrm{ax}}=3.75 \mathrm{~m}$ configuration, $D_{\mathrm{nc}}$ is negative enough to surpass the weakly destabilizing $D_{R}{ }^{\dagger}$. These numerical results seem to explain the observed tendency that the island "self-heals" more easily in the $R_{\mathrm{ax}}=3.75$ m configuration than in the $R_{\mathrm{ax}}=3.6 \mathrm{~m}$ configuration. Granting the "self-healing" by the neoclassical effect, the reappearance of the island in Fig. 2(c) may be explained by the suppression of the bootstrap current due to high collisionality $(>20)$, but for qualitative argument we need more accurate theory and experiment. The observed "self-healing" property is a favorable feature of LHD compared with the tokamak, in which a seed island grows as well known as neoclassical tearing instability [17].

The behavior of the island in response to pellet injection is diverse, as mentioned above. This may be explained by the $C$ term in Eq. (1), which can be regarded as an internal error field, with $s= \pm 1$ being replaced with $s=\exp \left(i \phi_{B}\right)$ to allow the arbitrary phase relation between external and internal error fields. Upon pellet injection, global plasma current may be induced and may generate an internal error field that resonates on a rational surface, which may differ in magnitude and phase depending on the plasma parameters when the pellet is injected, causing the island to appear diversely as observed. The pellet injection may also alter the sign and magnitude of $D$ appreciably, thus causing the phenomenon to be extremely complex.

One of us (Narihara) acknowledges discussions with Professor K. Itoh and Professor T. Hayashi. He also thanks Professor Y. Hamada for his repeated encouragement.

[1] R. D. Hazeltine and J.D. Meiss, Plasma Confinement (Addison-Wesley, Reading, MA, 1992).

[2] M. Wakatani, Stellarator and Heliotron Devices (Oxford University Press, New York, 1998).

[3] J. R. Cary and M. Kotschenreuther, Phys. Fluids 28, 1392 (1985).

[4] C. C. Hegna and A. Bhattacharjee, Phys. Fluids B 1, 392 (1989).

[5] T. Hayashi, A. Takei, and T. Sato, Phys. Fluids B 4, 1539 (1992).

[6] T. Hayashi, T. Sato, P. Merkel, J. Nührenberg, and U. Schwenn, Phys. Plasma 1, 3262 (1994).

[7] A. Bhattacharjee, T. Hayashi, C. C. Hegna, N. Nakajima, and T. Sato, Phys. Plasmas 2, 883 (1995).

[8] C. C. Hegna, Report No. UW-CPTC 99-6, 1999.

[9] R. Jaenicke et al., Nucl. Fusion 33, 687 (1993).

[10] S. Sudo et al., Rev. Sci. Instrum. 72, 483 (2001).

[11] O. Motojima et al., Phys. Plasmas 6, 1843 (1999).

[12] M. Fujiwara et al., Plasma Phys. Controlled Fusion 41, B157 (1999).

[13] N. J. Lopes Cardozo et al., Plasma Phys. Controlled Fusion 39, B303 (1997).

[14] M. F. F. Nave et al., Nucl. Fusion 32, 825 (1992).

[15] K. Narihara, I. Yamada, K. Yamauchi, and H. Hayashi, Rev. Sci. Instrum. 72, 1122 (2001).

[16] K. Watanabe, N. Nakajima, M. Okamoto, Y. Nakamura, and M. Wakatani, Nucl. Fusion 32, 1499 (1992).

[17] Z. Chang et al., Phys. Rev. Lett. 74, 4663 (1995). 